visual hallucinosis are typical of the amphetamine psychosis first described by Connell (Maudsley Monograph 1958). This problem is a seriously underreported side-effect of such drugs, and highlights the need for extreme caution in their use.

Deborah BROOKE

ROBERT KERWIN KEITH LLOYD

The Maudsley Hospital

Denmark Hill

London SE5 8AZ

The Place of Benzodiazepines in Psychiatric Practice

SIR: I am concerned that, in spite of the provisos mentioned, the paper on benzodiazepines by Tyrer \& Murphy (Journal, December 1987, 151, 719-722) will, in effect make a further contribution towards inhibiting the judicious and careful use of these drugs in appropriate cases based on experienced clinical judgement. As the authors state, patients are already "being encouraged to sue doctors for making them dependent" (on benzodiazepines). A sense of proportion is surely required here.

There are still many patients with chronic anxiety symptoms who do not respond to expertly applied alternative therapeutic techniques and, especially if "over $50 \%$ can stop their medication without withdrawal problems" and "... from present evidence there is no unequivocal permanent handicap caused by benzodiazopines in short or long-term dosage", it seems to me that clinical psychiatrists should have the courage to publicise these points in a responsible manner so that the media in particular and the public in general are better informed on these important problems.

Our Lady of Compassion Hospital

Maurice Silverman

Preston New Road

Blackburn BB2 7BG

SIR: Our comment about the medical and legal implications of prescribing benzodiazepines was made to draw psychiatrists' attention to the image of benzodiazepines portrayed in the media. If patients are going to sue doctors for prescribing benzodiazepines, the profession needs to be appraised of this fact, if only to ensure that adequate records are kept of consultations involving drug prescription. Currently we know of some $\mathbf{4 0 0}$ patients in the United Kingdom who have approached solicitors because of problems they have had with benzodiazepines. We agree with Dr Silverman that it is right to draw attention to the benefits as well as the risks of benzodiazepines, but if the use of these drugs is injudicious and careless the consequences could be serious.

PETER TYRER

Mapperley Hospital

SIOBHAN MURPHY

Porchester Road

Nottingham NG3 6AA

\section{Neurosyphilis and Psychiatry}

SIR: In their recent report of a patient with neurosyphilis, Brooke et al (Journal, October 1987, 151, 556) stress the importance of serological screening tests for syphilis in psychiatric patients whose mental state suggests an organic component. We have reviewed the cases of neurosyphilis presenting to the psychiatric unit of Tygerberg Hospital, the results of which serve to emphasise this view. Of 4314 consecutive admissions to the psychiatric unit between January 1983 and June 1987, 53 had a positive cerebrospinal fluid treponemal antibody absorption test. Thirty-two ( $0.74 \%$ of all admissions) satisfied criteria (Burke \& Schaberg, 1985) for a diagnosis of neurosyphilis; of these, 16 were suffering from acute, treatable psychiatric conditions, namely delirium $(n=6)$, mania $(n=5)$, hallucinosis $(n=4)$, and depression $(n=1)$. These data suggest that, especially in developing countries such as South Africa, routine serological tests for syphilis in psychiatric patients remain essential.

R. A. EMSLEY

M. C. ROBERTS

E. A. Higson

University of Stellenbosch

J. J. F. TALJAARD

PO Box 63

Tygerberg 7505

South Africa

Reference

BURKe, J. M. \& SCHABerg, D. R. (1985) Neurosyphilis in the antibiotic era. Neurology, 35, 1368-1371.

\section{Heterogeneity in Sporadic Schizophrenia}

SIR: Lewis et al (Journal, September 1987, 151, 306-314) have recently reviewed their original hypothesis (Murray et al, 1985) that the presence or absence of a family history identifies subgroups of greater aetiological homogeneity within schizophrenia. While their own data on ventricle brain ratios (VBR) in schizophrenic patients without a family history of major psychosis being larger than those with a family history (Reveley et al, 1984) are in support, results from other studies are not consistent 